\title{
Approach of Non-linear Programming for Cost Optimization of Plane Truss System
}

\author{
Gülçağ Albayrak and Uğur Albayrak
}

\begin{abstract}
Optimization is a systematic effort made to improve cost-effectiveness and obtain the best results under given circumstances. Cost optimization can be defined as the achievement of reduction in the unit cost of services provided without damaging their suitability. Nowadays, research on cost optimization on structural engineering is a developing area. Some convenient optimization methods can be effective for engineers to find the most cost-effective solutions for sizing of structural members. Furthermore, designing and producing of truss systems which are increasingly popular are quite appropriate with optimization. This paper discusses using non-linear programming to optimize cost of plane truss. The aim of the study is to obtain the minimum cost based on the optimal configurations which determined by mathematical modelling. The decision variables of the mathematical model are considered cross-sections of the truss system elements which are constrained in regard to structural and design issues. Also, the cost expression as an objective function is defined using cost data. For this purpose, firstly, the proposed method is introduced in details. Then a numerical example is developed and analysed according to problem data and the frame of the non-linear programming principles. The results are evaluated and the effectiveness of the proposed optimization method is mentioned.
\end{abstract}

Index Terms - Cost optimization, non-linear programming, optimization methods, truss systems.

\section{INTRODUCTION}

Cost optimization is used for optimizing cost and requirements at the same time to determine the variables which are both cost-effective and suitable. The design variables that produce the best results are usually the most expensive to do. Cost optimization determines a compromise between minimizing cost and satisfying the requirements.

Cost is one of the most important components of the structural design. Modelling the most economical buildings providing sufficient safety against collapse is always targeted by engineers. The selection of the most economical construction system depends on loads determined the minimal cross-sections. Especially truss systems are suitable for this generalization due to their uniformity. Trusses are simple structures widely used in civil engineering and other technical disciplines.

Truss system is preferred in a broad category of the structures, mostly made of steel, including bridges, towers, cranes, roofs and construction frameworks. Trusses derive

Manuscript received February 13, 2017; revised June 1, 2017.

Gülçağ Albayrak and Uğur Albayrak are with Eskisehir Osmangazi University, Civil Engineering Dept., Eskisehir, Turkey (e-mail: galbayrak@ogu.edu.tr, albayrak@ogu.edu.tr). their utility and strength from their simple construction: rod elements (bars) which exert only axial forces, connected concentrically with welded or bolted joints [1].

In generally, the aim is to design the structural systems with minimum weight, minimum cost and maximum strength for constructions.

To decide the most suitable structural configuration, engineers take advantage of their experience as well as try and error method. However numerous variables, lots of constraints and nonlinear behaviour of the structural members make the problem complex and time consuming to be solved.

Consequently, modelling of these structures by hand traditionally can be difficult, herewith; an automated method of generating the solutions has been desired. For this reason, some optimization methods were suggested by researchers in the literature.

Various studies can be found on the optimization of truss systems. Many of them are related with structural optimization such as shape and topology based optimization methods. Some early studies can be summarized as follows: Rajeev and Krishnamoorthy [2], Deb and Gulati [3] and Shrestha and Ghaboussi [4] have studied the use of genetic algorithms. Hansen and Vanderplaats [5] proposed an approximation method based on Taylor series expansion of the member forces. Elperin [6] suggested a Monte Carlo annealing algorithm. Also Papadrakakis et. al [7] studied with evolutionary strategies.

On the other hand, only a few studies considered cost optimization with different mathematical methods and software based models. For example, Jarmai et al. [8] considered cost optimization of a welded box beam and stiffened plate in their work. Also, Farkas and Jarmai [9] present a detailed cost calculation method for steel structures and optimize multi-types of steel frames for structural costs considering seismic effects.

Further examples can be mentioned: Kravanja and Zula [10] studied cost optimization of industrial frame structures.

Balogh and Vigh [11] aimed to find optimum steel configurations with minimum structural cost in their study. Optimization techniques, which are based on an optimality criteria approach, mathematical programming is widely employed [12].

Some of the studies on the optimization of trusses obtained from literature survey are shown in Table I.

The purpose of the study presented in this paper is to propose non-linear programming method for cost optimization of the plane truss. As well as in the similar works, the aim is to minimize the weight of the structure subject to constraints on design, stress and displacements for calculating the minimum cost. 
TABLE I: SELECTED STUDIES IN THE LITERATURE ABOUT THE OPTIMIZATION OF TRUSSES

\begin{tabular}{|c|c|c|}
\hline Author & Problem & Objective \\
\hline Gil and Andreu [13] & Non-parallel chords & $\begin{array}{l}\text { Stress and } \\
\text { geometric }\end{array}$ \\
\hline Kripakaran et. al. [14] & $10,18,21$-bar system & Minimum cost \\
\hline Silih and Kravanja [15] & Non-parallel chords & $\begin{array}{l}\text { Minimum mass } \\
\text { or cost }\end{array}$ \\
\hline $\begin{array}{c}\text { Farkas and Jarmai } \\
{[16]}\end{array}$ & $\begin{array}{l}\text { Simply supported } \\
\text { parallel chords }\end{array}$ & $\begin{array}{l}\text { Optimum height, } \\
\text { minimum volume }\end{array}$ \\
\hline
\end{tabular}

On the other hand, none modifications of the geometric model are allowed. Decision variables influence neither the topology nor the geometry of the truss which are considered to be fixed. The problem concerns a modification of cross-sections of the structural elements.

\section{METHOD}

Non-linear programming is the process of solving a mathematical model of equalities and inequalities, collectively termed constraints, over a set of unknown variables, along with an objective function to be maximized or minimized, where even if one of the constraints or the objective function is non-linear [17].

Truss optimization problem with regard to cost can be solved by the formulation of constraint functions and by the minimization of an objective function considering constraints.

Firstly, the mathematical model should be defined to solve these types of minimization problems effectively.

The mathematical model derived truss optimization problem includes three components: Design constraint, fabrication constraint and cost function. Thus, this model assures to satisfy all the important engineering requirements: safety, manufacturability and reasonability.

A cost function and constraints on design and fabrication should be mathematically formulated in the function of variables. At the level of synthesis the cost function should be minimized using effective mathematical methods for the constrained function minimization.

Comparing the optimum solutions designers can select the most suitable one. This comparison can result in significant mass and cost savings in the design stage.

The general formulation of a single-criterion non-linear programming problem is as follows where $f(x)$ is a multivariable non-linear function, $g_{j}(x)$ and $h_{i}(x)$ are non-linear inequality and equality constraints, respectively. All the functions $f(x), g_{j}(x)$ and $h_{i}(x)$ must be continuous and differentiable.

$$
\text { minimize } f(x) \quad, x=\left\{x_{1}, x_{2}, \ldots, x_{N}\right\}
$$

which subject to:

$$
\begin{array}{cl}
g_{j}(x) \leq 0 & j=1,2, \ldots, P \\
h_{i}(x)=0 & i=P+1, \ldots, P+M
\end{array}
$$

In view of the optimization of composite trusses, the continuous variables define dimensions, forces, stresses, strains, cost parameters, etc. The (in)equality constraints and the bounds of the variables represent a rigorous system of design, load, resistance and deflection functions known from the structural analysis. The objective function is proposed to minimize the structure's manufacturing costs.

This research deals with cost optimization based on non-linear programming. The aim of the study is to minimize the cost of truss formulated as the objective function through minimized cross-section of the truss elements. For this reason, the truss mass as well as cost is minimized for each bar to obtain the optimum cross section area.

Mathematical expressions of the general approach can be seen below. $A_{i}$ indicates the cross-section area of the $i^{\text {th }}$ which minimizes the weight of the system. $\mathrm{C}$ is the unit cost of the truss material (steel generally).

Thus, this optimization problem with constraints can be formulated as follows:

Minimize the total cost

$$
\Sigma=\Sigma \rho_{i} \cdot A_{i} \cdot L_{i} \cdot C \text { with } i=1,2, \ldots, n
$$

which subject to:

$$
\begin{array}{ll}
A_{\text {min }} \leq A_{i} \leq A_{\text {max }} & \text { for } i=1, \ldots, n \\
\sigma_{\text {min }} \leq \sigma_{i} \leq \sigma_{\text {max }} & \text { for } i=1, \ldots, n \\
u_{\text {min }} \leq u_{j} \leq u_{\text {max }} & \text { for } j=1, \ldots, k
\end{array}
$$

where $n$ is the number of bars of the truss, $\mathrm{k}$ is the number of nodes, $L_{i}$ is the length of the $i^{\text {th }}$ bar, $\rho_{i}$ is density, and $A_{i}$ is cross-section area of the $i^{\text {th }}$ bar. $u_{j}$ is the nodal vertical displacement of node $j$.

\section{NUMERICAL EXAMPLE}

The simply supported plane truss which has parallel chords and ten bars designed as Figure 1. The geometrical properties, loading and boundary conditions of the truss are shown in Fig. 1. The system is loaded on 2 nodes with $500 \mathrm{kN}$ vertically. The cross-section areas of the bars are assigned as decision variables in this problem. Each bar has been limited with maximum stress constraint of $200 \mathrm{MPA}$ and minimum area of $100 \mathrm{~mm}^{2}$. Other numerical data is following: $\mathrm{a}=2.5 \mathrm{~m}, \mathrm{E}=$ $2.1 \times 10^{5} \mathrm{MPa}, \rho=7.85 \times 10^{-6} \mathrm{~kg} / \mathrm{mm}^{3}, \mathrm{~F}=500 \mathrm{kN}$., $u_{\text {allow. }}= \pm 50$ $\mathrm{mm}$. Material cost $=1.50 \$ / \mathrm{kg}$.

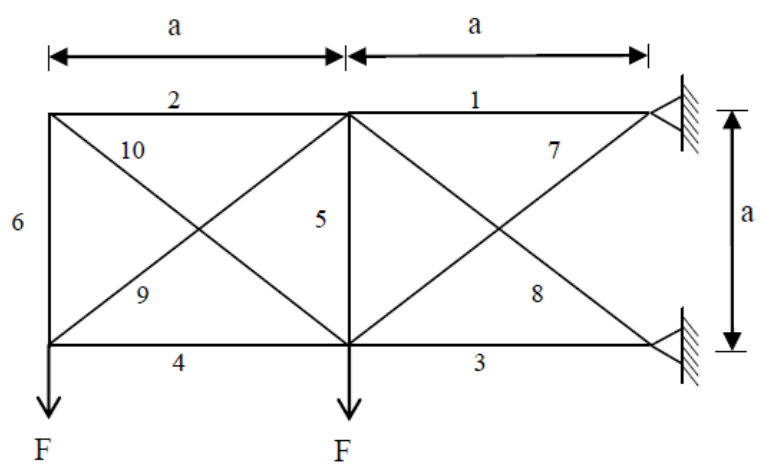

Fig. 1. Truss cantilever with ten bars

$$
\begin{gathered}
u_{j}=\Sigma\left(P_{i} P_{i}{ }^{\prime} / E A_{i}\right) L_{i} \quad i=1,2, \ldots, 10 . \\
I_{i}=\pi^{2} r_{i}^{4} / 4
\end{gathered}
$$


TABLE II: OPTIMIZATION RESULTS OF THE NON-LINEAR PROGRAMMING

\begin{tabular}{cc}
\hline Bar No. & $\begin{array}{c}\text { Cross-Sectional } \\
\text { Area }\left(\mathrm{mm}^{2}\right)\end{array}$ \\
\hline 1 & 9245.14 \\
2 & 100.00 \\
3 & 6419.34 \\
4 & 4148.56 \\
5 & 100.00 \\
6 & 254.83 \\
7 & 1468.45 \\
8 & 5496.74 \\
9 & 5550.16 \\
10 & 100.00 \\
\hline Weight $(\mathrm{kg})$ & 7522.52 \\
Cost $(\$)$ & 11283.78 \\
\hline
\end{tabular}

where $u_{j}$ is the vertical displacement of the node $j, P_{i}$ and $P_{i}$ ' are the bar forces of the $i^{\text {th }}$ bar, $\mathrm{E}$ is the modulus of elasticity, $\mathrm{A}_{\mathrm{i}}$ and $\mathrm{L}_{\mathrm{i}}$ are the cross-sectional area and length of $i^{\text {th }}$ bar, respectively. $I_{i}$ is the moment of inertia and $r_{i}$ is the radius of the $i^{\text {th }}$ bar.

$$
f(x)=C \rho \Sigma A_{i} L_{i}
$$

$f(x)$ is the objective function minimized by decision variables. The objective function considered is the total cost of the structure subjected to material and performance constraints in the form of stress and deflection limits.

The radius of the bars are assigned as decision variables $\left(r_{1}\right.$, $\left.r_{2}, \ldots, r_{10}\right)$.

In the optimization model includes inequality constraints which are considered as follows:

$$
\begin{aligned}
& \text { Tensile constraint } \quad: \sigma_{i} \leq \sigma_{\max } \\
& \text { Compression constraint }: P_{i} \leq\left(B_{c r}\right)_{i} \\
& \left(B_{c r}\right)_{i}=\pi^{2} E I_{i} / v L_{i}^{2}
\end{aligned}
$$

$\left(B_{c r}\right)_{i}$ is the critical buckling load where $\mathrm{v}=2.5$ is considered as the safety coefficient.

$$
\begin{aligned}
& \text { Displacement constraint: } u_{j} \leq u_{\max }=\mathrm{a} / 50 \\
& \text { Size constraints: } \mathrm{r}_{1}, \mathrm{r}_{2}, \ldots, \mathrm{r}_{10} \geq 5.65
\end{aligned}
$$

Consequently, this is a non-linear optimization problem with 4 constraints and 10 design variables.

The optimization problem formulated above is solved using the MATLAB optimization toolbox, which contains an extensive library of computational algorithms for solving different optimization problems, such as non-linear minimization. The library function "fmincon" solves optimization problems with objective functions under linear/non-linear constraints which are included mathematical models. MATLAB fmincon optimization function includes the following syntax:

$$
x=\text { fmincon(fun, } \mathrm{x} 0, \mathrm{lb}, \mathrm{ub}, \text { nonlcon,options })
$$

where fun denotes the scalar objective function of a multidimensional design vector $x$, while nonlcon contains non-linear non-equality $(c(x) \leq 0)$ constrained functions. LB and UB are vectors containing the lower and upper bounds of the design valuables and $\mathrm{x} 0$ is the initial design point.
In order to find the minimum mass and minimum cost, cross-section areas for each bar are calculated using "fmincon" tool as a library function in MATLAB.

The calculation results are given in Table II.

As shown in the Table II, the bar number of 2, 5, 10 have the minimum cross-sections. The weight of the structure was calculated $7522.52 \mathrm{~kg}$. According to the weight of the structure, total cost of the truss is found $\$ 11283.78$.

\section{CONCLUSION}

The aim of the research was to introduce an easy technique for solving cost optimization problems in plane truss systems. So in this paper, non-linear programming was proposed for finding an optimal solution with using MATLAB. The objective function involving the decision variables and structural constraints with non-linear functions were formed to mathematical model of the problem.

A numerical example was executed to illustrate the process of proposed method. Thus, minimum cost of the cantilever truss structure was obtained. The cost function expresses the cost of material according to the weight of the structure. Therefore, it was shown that non-linear programming, as an optimization technique, can be an effective tool for solving these types of problems with minimum user effort and time. Essentially, the proposed method can be applied effectively on each type of the truss models through deciding on the unknown variables and substituting them into the related constraints.

With some modifications, the suggested method may also be used in the space truss systems as well as plane truss systems.

Future works can be concerned with the continuation of the validation (for more complex problems) and comparison with other optimization methods such as metaheuristic algorithms to choose the best numerical technique for these types of problems.

\section{REFERENCES}

[1] J. Smith , J. Hodgins , I. Oppenheim, and A. Witkin, "Creating models of truss structures with optimization," ACM Transactions on Graphics (TOG), vol. 21, no. 3, pp. 295-301, 2002

[2] S. Rajeev and C.S. Krishnamoorthy, "Genetic algorithms-based methodologies for design optimization of trusses," Journal of Structural Engineering, pp. 350-358, 1997.

[3] K. Deb and S. Gulati, "Design of truss-structures for minimum weight using genetic algorithm," Finite Elements in Analysis and Design, vol. 37, pp. 447-465, 2001

[4] S. M. Shrestha and J. Ghaboussi, "Evolution of optimum structural shapes using genetic algorithm," Journal of Structural Engineering, pp. 1331-1338, 1998.

[5] S. R. Hansen and G. N. Vanderplaats, "Approximation method or configuration optimization of trusses," AIAA Journal, vol. 28, no. 1, pp. 161-168, 1990.

[6] T. Elperin, "Monte-Carlo structural optimization in discrete variables with annealing algorithm," International Journal of Numerical Methods in Engineering, 26, pp. 815-821, 1988.

[7] M. Papadrakakis, N. D. Lagaros, G. Thierauf, and J. Cai, "Advanced solution methods in structural optimization based on evolution strategies," Engineering Computations, vol. 15, no. 1, pp. 12-34, 1998.

[8] K. Jarmai, J. Farkas, and P. Uys, "Optimum design and cost calculation of a simple frame with welded or bolted corner joints," Welding in the World, vol. 48, no. 1-2, pp. 42-49, 2004.

[9] J. Farkas and K. Jarmai, Optimum Design of Steel Structures, Springer, p. 265.2013. 
[10] S. Kravanja and T. Zula, "Cost optimization of industrial steel building structures," Advances in Engineering Software, vol. 41, pp. 442-450, 2010.

[11] T. Balogh and L. G. Vigh, "Cost optimization of concentric braced steel building structures," World Academy of Science, Engineering and Technology, vol. 7, pp. 1016-1025, 2013.

[12] W. Kuntjoro and J. Mahmud, "Truss structural configuration optimization using the linear extended interior penalty function method," Anzıam Journal, vol. 46, pp. 1311-1326, 2005.

[13] L. Gil and A. Andreu, "Shape and cross-section optimization of a truss structure," Computers and Structures, vol. 79, pp. 681-689, 2001.

[14] P. Kripakaran, A. Gupta, and J. W. Baugh, "A novel optimization approach for minimum cost design of trusses," Computers and Structures, vol. 85, pp. 1782-1794, 2007.

[15] S. Silih and S. Kravanja, "Topology, shape and standard sizing optimization of trusses using MINLP optimization approach," Design, Fabrication and Economy of Welded Structures International Conference, Miskolc, Hungary, 2008.

[16] J. Farkas and K. Jármai, Design and Optimization of Metal Structures, Horwood Publishing, Chichester, pp. 143-150, 2008.

[17] M. Asadujjaman and M. Babul Hasan, "A proposed technique for solving quasi-concave quadratic programming problems with bounded variables," Dhaka University Journal of Science, vol. 63 no.2, pp. 111-117, 2015.

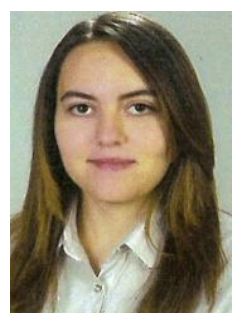

Gülçağ Albayrak received her B.Sc in civil eng, a $\mathrm{M} . \mathrm{Sc}$ in construction management and a $\mathrm{PhD}$. in construction management from Eskisehir Osmangazi University (ESOGU) Dept. of Civil Engineering, Turkey. She is working as a research assistant in Construction Management Division of ESOGU Civil Eng. Dept. Her research interests include value engineering applications, statistical evaluations, engineering management, mathematical modelling, metaheuristic methods and optimization techniques in civil engineering.

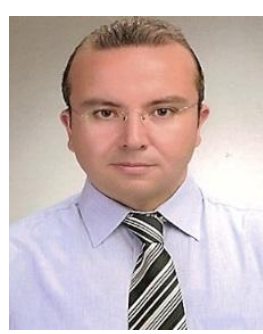

Uğur Albayrak received his B.Sc. in civil engineering, a M.Sc in structural engineering and a PhD. in structural engineering from Eskisehir Osmangazi University (ESOGU) Dept. of Civil Engineering, Turkey. He is presently working as an assistant professor at the same department. His main research interests include design of steel structures, R.C. structures, earthquake resistant design and computer applications in Civil Eng., and having more than 10 publications in national and international journals. 